\title{
Hypomethylation of tissue factor pathway inhibitor 2 in human placenta of preeclampsia
}

\author{
Xirong Xiao ${ }^{1}$, Xiang Tao ${ }^{1}$, Yongxiang $\mathrm{Wang}^{2}$, Lisha Zhu ${ }^{3}$, Yunzhen $\mathrm{Ye}^{1}$, Haiyan Liu ${ }^{1}$,
}

$$
\text { Qiongjie Zhou }{ }^{1}, \text { Xiaotian } \mathrm{Li}^{* 145} \text {, Yu Xiong*145 }
$$

${ }^{1}$ Obstetrics and Gynecology Hospital of Fudan University, Shanghai 200032, China

${ }^{2}$ Department of Obstetrics and Gynecology, Shuguang Hospital of Shanghai University of Traditional Chinese Medicine, Shanghai 201203, China

${ }^{3}$ The International Peace Maternity \& Child Health Hospital of China Welfare Institute, Shanghai 200032, China

${ }^{4}$ Key Laboratory of Molecular Medicine, Ministry of Education, Shanghai Medical College, Fudan University, Shanghai 200032, China

${ }^{5 .}$ Institute of Biomedical Sciences of Fudan University, Shanghai 200032, China

* Corresponding Authors

Yu Xiong and Xiaotian Li are to be contacted at the Obstetrics and Gynecology Hospital of Fudan University, 128 Shenyang Road, Shanghai, 200090, China; Tel.: +86 21 33189900; Fax: +86 21 63455090.

E-mail addresses:

Yu Xiong: xiongyu1535@163.com

Xiaotian Li: xiaotianli555@163.com 


\begin{abstract}
Objectives: To investigate the expression, DNA methylation status and its regulatory mechanism of tissue factor pathway inhibitor 2 (TFPI-2) in human placenta tissues of preeclampsia (PE).
\end{abstract}

Material and methods: We studied the mRNA and protein expression and the promoter methylation levels of TFPI-2 in the PE placentas compared with those in the normal pregnant (NP) women. Quantitative real-time polymerase chain reaction, immunohistochemistry, western blot, and Sequenom MassARRAY were used for placenta tissue detection.

Results: The expressions of TFPI-2 mRNA and protein were significantly elevated in the PE placentas when compared with those in the NP ones $(P<0.05)$.

Hypomethylation of the TFPI-2 promoter was detected both in PE patients and NP women, with a significant decrease in $\mathrm{PE}$ placentas $(\mathrm{P}=0.005)$. The methylation level was significantly decreased at CpG_6 (-168 to -167$),$ CpG_15 (-98 to -97) and CpG_18.19 (-68 to -65$)$ in $\mathrm{PE}$ patients than that in normal placentas $(\mathrm{P}<0.05)$. However, the expression of DNMT-1 didn't show significant difference between the two groups $(\mathrm{P}>0.05)$.

Conclusion: Over-expression of TFPI-2 and aberrant promoter mythylation status presented in the PE placentas, suggesting that epigenetic mechanism might contribute to the pathogenesis of PE.

Keywords: DNMT-1, methylation, placenta, preeclampsia, TFPI-2. 
Abbreviations list: TFPI-2, tissue factor pathway inhibitor 2; PE, preeclampsia; DNMT, DNA methyltransferase enzymes; NP, normal pregnant; BMI, body mass index; MAP, mean arterial pressure; LSD, lysine-specific demethylase 


\section{Introduction}

Preeclampsia (PE), affecting pregnancy by $3-5 \%$, is a pregnancy-specific syndrome routinely diagnosed with the combined presentation of high blood pressure and proteinuria [1]. PE is one of the main causes of maternal, fetal and neonatal mortality, especially in developing countries [2]. Although a great deal of research has been conducted to explore the precise pathogenesis, the complicated molecular mechanism of PE development and progression remains unclear. Accumulating evidence supports the hypothesis that thrombosis, accompanied with many changing coagulation factors such as tissue factor (TF) and tissue factor pathway inhibitor (TFPI), is an important contributing factor of PE development [3-5].

The tissue factor pathway inhibitor 2 (TFPI-2), located on chromosome $7 \mathrm{q} 22$, is a 32-kDa Kunitz-type serine proteinase inhibitor [6, 7]. The human TFPI-2 gene, expressed abundantly in full-term placenta and widely in a variety of adult human tissues such as liver, skeletal, muscle, heart, kidney and pancrease, has also been shown to reduce tumor invasion and metastasis [8]. TFPI-2 may play an important role in pregnancy maintance, because the serum TFPI-2 can extremely increase during pregnancy and return to prepregnancy level several days after delivery, as evidenced by our previous report that demonstrated a significantly increased expression of TFPI-2 in PE placenta based on immunohistochemical analysis [9]. Expression of TFPI-2 inhibited migration, proliferation and invasion in Bewo and in JEG-3 cell lines, which may contribute to the pathogenesis of PE [10]. However, the molecular mechanisms regulating the differential placental expression of TFPI-2 have not been 
identified.

DNA methylation of the imprinted genes is an intriguing mechanism linking environmental cues to placental pathology [11]. Aberrant DNA methylation has been demonstrated to control multiple physiological and pathological processes in trophoblast and placenta $[12,13]$. As an imprinted gene cluster, the promoter of TFPI-2 has been reported to contain a CpG island region of 220 bp that spans exon1 and three transcription initiation sites, associated with transcriptional silencing of the gene [14-16]. Studies have also shown that TFPI-2 is frequently inhibited by promoter hypermethylation in non-small-cell lung cancer, gastric stromal tumor, cervical cancer and nasopharyngeal carcinoma [8, 17-19]. The development of placenta involves proliferation, invasion and migration of placental trophoblasts into the myometrium of the uterus, in a way that is similar to that in most malignant tumors [20]. In light of the close resemblance between the process of tumorigenesis and placental development, we hypothesized that promoter methylation could be the possible regulatory mechanism for the expression of TFPI-2.

To explore the role of methylation in regulating the differential expression of TFPI-2, we investigated the expressions of TFPI-2 mRNA and protein, and evaluated the methylation status of TFPI-2 in the human placenta tissues from PE patients and normal pregnancy. To date, there have been no such reports, as indicated by our literature review, on the methylatic mechanism regulating the TFPI-2 expression involved in the development of PE. 


\section{Material and Methods}

\section{Study participants}

A total of 29 placenta samples, 10 diagnosed with normal pregnancy and 19 with PE, were obtained from the pregnant women attending the Obstetrics and Gynecology Hospital of Fudan University from July 2014 to June 2015. The diagnosis was made of PE according to the criteria of ACOG 2013: onset of hypertension in a previously normotensive woman and proteinuria (at least $0.3 \mathrm{~g}$ of protein in a 24-hour urine sample) [21]. The cases diagnosed as chronic hypertension, diabetes mellitus, chronic nephritis and systemic lupus erythematosis were excluded. All of the participants, who were of Han ethnicity, received cesarean section. The women in the group of normal pregnancy chose cesarean delivery according to their personal request or had a history of uterine scar. The current study adhered to the principles of the Declaration of Helsinki and was approved by the institutional ethics committee. Written informed consent was obtained from all participants.

\section{Sample collection and preparation}

After placentas birth, three blocks $(1 \times 1 \times 1 \mathrm{~cm})$ of chorionic villi were dissected from the central cotyledon on the maternal surface of each subject. When the samples were washed with phosphate buffered saline (PBS), one sample block was fixed in $4 \%$ formalin, and the other two blocks were frozen in liquid nitrogen to be stored at $-80^{\circ} \mathrm{C}$

\section{Immunohistochemistry}

Formalin-fixed and paraffin-embedded, the samples of the placenta tissues were 
studied. The 4- $\mu$ m-thick tissue sections were deparaffinized and rehydrated before immersed in EDTA buffer and subjected to antigen microwave retrieval. The sections were incubated with rabbit monoclonal anti-human TFPI-2 (Abcam, USA; dilution 1:100), DNMT-1 (Abcam, USA; dilution 1:100), DNMT-3a (Abcam, USA; dilution 1:100) or DNMT-3b (Abcam, USA; dilution 1:100) individually at $4^{\circ} \mathrm{C}$ overnight in a humidified chamber. Immunostaining for TFPI-2 and DNMTs was then revealed using a standard streptavidin-biotin peroxidase complex kit with diaminobenzidine as a chromogen (Tiangen Biotech, China). The negative controls were established by omitting the primary antibody of the same species.

When the images were acquired with an Olympus BX50 microscope digital camera (Olympus, Japan), staining intensity of TFPI-2 was analyzed using the computerized image analysis software (Image-Pro® Plus v6.0 Cybernetics) [22-24]. As DNMTs were nuclei expressed, all the slides for DNMTs analysis were independently reviewed by two investigators (XR Xiao and X Tao). The nuclei were regarded as being stained specifically only when there was an agreement between the two observers. The stained nuclei were scored by estimating the percentage of the syncytiocytotrophoblast cells $[25,26]$.

\section{Quantitative real-time polymerase chain reaction (qRT-PCR)}

Total RNA was extracted from the placenta samples using Trizol (Invitrogen,

Carlsbad, CA) according to the manufacturer's protocols. The first-strand complementary DNA (cDNA) was synthesized with M-MLV reverse transcriptase (Promega, USA). The primer sequences for TFPI- 2 cDNA were $\left(5^{\prime} \rightarrow 3^{\prime}\right)$ : 
TTCTGCGCACCAAAGAAA (F), TCTTGGATTAAAATAATAGCGAGTCA (R). qRT-PCR were conducted to detect the relative mRNA levels using Power SYBR Green PCR Master Mix (Applied Biosystems, USA) on the Bio-Rad IQ5 Real Time PCR System (Bio-Rad, USA). Glyceraldehyde-3-phosphate dehygrogenase (GAPDH) was used as an endogenous control. The primer sequences for GAPDH cDNA were $\left(5^{\prime} \rightarrow 3^{\prime}\right)$ : TTCGACAGTCAGCCGCATCTT $(\mathrm{F})$, CCCAATACGACCAAATCCGTT (R). The results were calculated using $2-\Delta \Delta \mathrm{Ct}$.

\section{Western blot}

From the placenta tissues the total protein was extracted using radioimmuneprecipitationassay (RIPA) buffer containing $1 \mathrm{mmol} / \mathrm{L}$ protease inhibitor cocktail. Upon 5-min heating at $95^{\circ} \mathrm{C}, 20 \mu \mathrm{g}$ of denatured protein for each reaction was used to load a $12 \%$ polyacrylamide SDS for electrophoresis. Polyvinylidenedifluoride (PVDF) membranes were treated as the transfer by a wet transferring system. With 2-h blocking at room temperature, the membrane was probed with primary antibody (Abcam, USA; dilution 1:1000) at $4{ }^{\circ} \mathrm{C}$ overnight, and with secondary HRP-conjugated antibody for $1 \mathrm{~h}$ at room temperature. The immunoreactive bands were detected by chemiluminescence and photographed in ChemiDoc ${ }^{\mathrm{TM}}$ Imaging Systems. GAPDH was used as an endogenous protein for normalization. The blot was analyzed using Image $\mathrm{J}$ software, and the ratio of the absorbance volume was normalized to GAPDH.

\section{DNA preparation and bisulfite conversion}

To avoid the potential effects on DNA methylation of glucocorticoid, we studied the 
methylation status in $10 \mathrm{PE}$ patients and all 10 normal pregnancy. Genomic DNA was isolated from the placentas using a QIAamp DNA Mini Kit (Qiagen, Hilden, Germany) according to the manufacturer's instructions. An RNase step was included during DNA isolation to avoid the contamination by RNA. The concentration and purity of genomic DNA were measured with absorbance at 260 and $280 \mathrm{~nm}$ using a NanoDrop $^{\mathrm{TM}} 1000$ spectrophotometer (Thermo Scientific, Wimington, USA). The extracted DNA was bisulfite modified using an EZ DNA Methylation Kit (Zymo Research, Orange, CA, USA) according to the protocols recommended. When the sequencing results were confirmed, cytosine residues were converted by more than $99.0 \%$.

\section{Quantitative MassARRAY analysis of gene methylation status}

An analysis was made of quantitative DNA methylation with MassARRAY EpiTYPER (Sequenom, San Diego, CA, USA), combining the base-specific enzymatic cleavage with MALDI-TOF mass spectrometry, which is considered to be a highly accurate and sensitive approach to the quantitative analysis of DNA methylation at $\mathrm{CpG}$ islands [27]. The detailed primers were designed using the online software Epidesigner (at http://www.epidesigner.com/, Supplementary files: Table S1). The quantitative methylation data for each $\mathrm{CpG}$ site or unites of multiple $\mathrm{CpG}$ islands were analyzed with the EpiTYPER software (Sequenom). Strict quality control was ensured before analysis. The samples were discarded missing more than $30 \%$ of data points as the unreliable and the $\mathrm{CpG}$ units failing to produce data from more than $30 \%$ of the samples as the unreliable [28]. 


\section{Statistical analysis}

All statistical analysis was performed on SPSS (Version 19.0; IBM, New York, USA) and GraphPad Prism (Version 5.0; GraphPad Software Inc., San Diego, CA, USA). The quantitative data were presented as mean \pm standard deviations, and the methylation levels and DNMT-1 expression levels were classified as quartiles based on the distributions. All the quantitative data in the two independent groups were analyzed through Mann-Whitney $U$ test. The analysis of Spearman Rank correlation was performed to evaluate the correlations between the levels of TFPI- 2 protein expression and mean arterial pressure (MAP). All statistical analysis was two-sided and $P<0.05$ was considered to be statistically significant. 


\section{Results}

\section{Patient characteristics evaluation}

As indicated by the demographic and clinical characteristics of the study groups (Table 1), the maternal age and antepartum body mass index (BMI) were comparable between of the PE patients and the NP women, while the former had lower mean gestational age at delivery and mean birthweight than the latter. As expected, the levels of systolic BP $(\mathrm{mmHg})$, diastolic $\mathrm{BP}(\mathrm{mmHg})$ and proteinuria $(\mathrm{g} / 24 \mathrm{~h})$ were higher in the PE than in the NP group, respectively.

\section{TFPI-2 expression and localization in placentas}

As evidenced by the immunohistochemistry (IHC) assay to detect the expression and localization of TFPI-2 in the placenta samples with PE, the brown signal was observed mainly in the cytoplasm of the syncytiocytotrophoblast layers, but not in the villi interstitium (Fig.1A). The semi-quantitation of immunostaining showed the mean density was significantly higher in the PE than in the NP group (0.0978 vs. 0.0537 ; $\mathrm{P}=0.0002$; Fig.1B). According to the analysis on the correlation between MAP before cesarean section and the mean density of TFPI- 2 expression in the 29 samples, a significant association was observed between the TFPI-2 expression and MAP $(\mathrm{r}=0.677 ; \mathrm{P}<0.0001 ;$ Fig.1C).

The evaluation of the differential expression of TFPI-2 mRNA through the performance of qRT-PCR in the preeclamptic and normal placentas, upon GAPDH standardization, indicated that significantly higher levels of TFPI-2 expression were observed in the PE placentas than in the controls. Approximately, the level of TFPI-2 
mRNA was 4-fold higher in the former than in the latter (3.52 [0.80-10.44] vs. 0.86 [0.28-2.07]; median; $\mathrm{P}<0.01$; Fig.1D).

The results from the western blot performed on the PE and NP placentas to further confirm the expression of TFPI-2 protein were found to be consistent with those obtained using IHC and qRT-PCR. The level of TFPI-2 protein, measured by densitometric analysis, was nearly 1.5 -fold higher in the PE than in NP placentas (0.92 [0.50-1.55] vs. 0.6 [0.5-1.29]; median; $\mathrm{P}<0.05$; Fig.1F).

\section{Methylation profiles of TFPI-2 in preeclamptic placentas}

As indicated by the analysis of the methylation level of TFPI-2 promoter region sequences on the Sequenom MassARRAY platform, where one region with the highest percentage of CpG sites in the promoter of TFPI-2 (-272 to 96, nucleotide positions relative to the translation start sites) was analyzed, it was found that the amplicon contained $31 \mathrm{CpG}$ sites which could be divided into $15 \mathrm{CpG}$ unites, and hypomethylation of the TFPI-2 promoter was detected both in the PE and NP placentas (Fig.2A). The methylation level was significantly lower in the PE placentas, with a median value of $5.5 \%$ (interquartile range: IQR $4.44 \%-7.03 \%$ ), than in the controls, with a median value of $8.75 \%$ (IQR 7.05\%-10.18\%; P=0.005; Fig.2B).

In the evaluation of the methylation status of every $\mathrm{CpG}$ unit to investigate the methylation level at different $\mathrm{CpG}$ sites, an analysis was made of 12 available $\mathrm{CpG}$ unites containing $22 \mathrm{CpG}$ sites, and the results showed that the methylation level was different at each $\mathrm{CpG}$ unit, ranging from 0 to $47 \%$ (Fig.2C), and that the methylation status at CpG_6 (-168 to -167$), \mathrm{CpG}_{-} 15$ (-98 to -97$)$ and $\mathrm{CpG} \_18.19$ (-68 to -65$)$ was 
significantly decreased in the $\mathrm{PE}$ patients than in the normal women $(\mathrm{P}<0.05$; Fig.2D-F). The difference at the other $\mathrm{CpG}$ units was not significant between the groups $(\mathrm{P}>0.05)$.

\section{Expression of DNMTs in preeclamptic placentas}

In the evaluation of the expression of DNA methyltransferase enzymes (DNMTs) to investigate the potential mechanism regulating the methylation level in the placenta, IHC staining showed that DNMT-1 was mainly expressed in the nuclei of syncytiotrophoblasts. However, the semi-quantitation of immunostaining for DNMT-1 showed no significant difference between the groups, with a median value of $46.67 \%$ in the PE group relative to $28.33 \%$ in normal one (P>0.05; Fig.3A-B). As DNMT3a and DNMT3b were faintly or negatively expressed in the nuclei of syncytiotrophoblasts either in the PE or in the normal placentas, their roles on TFPI-2 expression were not evaluated in the current study (Fig.3C-D). 


\section{Discussion}

In the current study, we showed that the expressions of TFPI- 2 mRNA and protein were significantly elevated in the PE placentas when compared with those in the NP ones, and that hypomethylation presented in the promoter of TFPI-2, with a significant decrease in PE placentas, suggesting that TFPI-2 regulated by epigenetic mechanism might contribute to PE pathogenesis.

TFPI-2 regulates negatively the enzymatic activity of plasmin, trypsin and VIIa-tissue factor complex and consequently plays an important physiological role in blocking endothelial dysfunction initiated coagulation in normal pregnancy $[6,9]$. TFPI-2 may also inhibit trophoblast cell proliferation and tumor-related anginogenesis [29-31]. Over-expression of TFPI-2 could inhibit intervillous remodeling, normal decidualization and appropriate placentation when it played a role in the development of PE [32]. These observations provided a new evidence for the potential pathogenesis of PE.

The current study showed an elevation of TFPI- 2 mRNA and protein expression in the PE relative to the NP placentas, which confirmed our previously reported finding [9]. Moreover, it revealed a significant association between TFPI-2 protein expression and MAP, which is an important prognosis predictor in PE [33]. These findings were consistent with those reported by Erez et al and Dusse et al [34, 35] but inconsistent with those revealed by Ogawa et al and Chinni et al [36, 37]. Several possible factors might account for these differences: TFPI-2 elevation could be related to the severity of PE; coordinated with the higher blood pressure, TFPI-2 could be 
strongly induced from the vascular smooth muscle cells exposed to the higher fluid shear stress in the placenta [38]; and the expression of TFPI-2 could be heterogeneous and unstable in different individuals due to varied coagulation status, diet and local environment.

There is an increasing body of evidence showing that epigenetic features are involved in the pathogenesis of PE; however, the role of DNA methylation of TFPI-2 in placenta dysfunction has not been explored. The current study showed that hypomethylation of the TFPI-2 promoter region was detected both in the PE and in the NP group, and that the general level of methylation decreased more significantly in the former than in the latter, which is consistent with many other studies on other diseases [14, 39-41].

However, not all hypomethylation status was inversely correlated with transcription of the gene. It was demonstrated that methylation of $\mathrm{CpG}$ sites downstream of transcription initiation site couldn't inhibit elongation in mammalian cells [42]. TFPI-2 promoter was reported to have 3 transcription initiation sites, and in the current study the hypomethylated $\mathrm{CpG}$ sites were found to be all at the upstream or nearby the major transcription initiation sites at position -75 upstream from the translation start site [39]. Hube et al revealed that unmethylated $\mathrm{CpG}$ sites between -112 and -63, where $\mathrm{CpG}_{-} 15$ (-98 - to -97$)$ and $\mathrm{CpG}_{-} 18.19$ (-68 to -65$)$ were located in the current study, allowed TFPI-2 transcription in human choriocarcinoma cells [39]. Dong et al demonstrated that $\mathrm{CpG} \_15$ and $\mathrm{CpG} \_18.19$ of TFPI-2 were more significantly hypermethylated in invasive cervical carcinomas than in cervical 
intraepithelial neoplasm tissues and healthy controls, which was consistent with the finding in the current study [19]. As indicated by our literature review, this is the first study showing the aberrant methylation status at CpG_6 (-168 to -167) of the TFPI-2 protmoter. In view of this, we hypothesized the methylation status at the three hypomethylated $\mathrm{CpG}$ sites could regulate the expression of TFPI-2.

Normally, DNA methylation is orchestrated by DNMTs. DNMT-1 was reported to be the predominant DNA methylating enzyme and maintain the DNA methylation patterns during DNA replication [43]. Unexpectedly, DNMT-1 was highly expressed both in the PE and the NP group and no significant difference was observed between the two groups, which was consistent with the finding previously reported [44]. This might be explained by the evidence that the methylation status of TFPI- 2 in the placenta could be regulated by some other factors. Actually, it was revealed that genomic hypomethylation didn't require DNMT-1 down-regulation at all [45]. Recent studies have shown that histone methylation plays an important role in the establishment of DNA methylation. Ribarska et al. showed that enrichment of histone modification presented at TFPI-2 promoter [14]. More effort is merited to get a deeper insight into the mechanism in the regulation of TFPI-2 expression and promoter methylation in placenta.

As one of the limitations in the current study, the gestation age was younger in the PE than in the control group. In our previous study, we had revealed that TFPI-2 increased steadily with gestational age and reached a peak at the $39^{\text {th }}$ gestational week with similar changes in placenta [9]. The current study showed that the expression of 
TFPI-2 protein in the PE group was nearly two times of that in the NP group at a smaller gestation, which indicated that the elevation of TFPI-2 could play an important role in PE pathogenesis. Additionally, the sample size was limited, which may affect the precision for large populations.

In conclusion, the current study confirmed the potential role of TFPI-2 in the pathogenesis of PE. Aberrant DNA methylation of TFPI-2 promoter, especially the methylation status at $\mathrm{CpG} \_6$ (-168 to -167$), \mathrm{CpG}_{-} 15$ (-98 to -97$)$ and $\mathrm{CpG}_{-} 18.19$ (-68 to -65), could regulate the expression of TFPI-2. However, the expression of DNMT-1 didn't show any significant difference between the two groups, which suggests other potential mechanisms involved in regulating DNA methylation of TFPI-2 promoter. Such findings should provide a heuristic basis for further studies on the role of histone methylation in placenta tissues and cell lines.

\section{Financial support}

This work was partly supported by the grants from the National Science Fund of China (81270712), National Science Foundation for Young Scholars of China (81200449), National Science Foundation for Young Scholars of China (81300506), National Science Foundation for Young Scholars of Shanghai (13ZR1452000), Health industry special funds for Public Benefit Research Foundation from the Ministry of Health, Special Fund for scientific Research in the Public Interest (201402006), National Key Basic Research Plan of China (973 Plan) (2015CB943300), the 
Shanghai Key Laboratory of Female Reproductive Endocrine-Related Diseases, Shanghai Key Laboratory of Birth Defects and the Key Specialty Project of the Ministry of Health, People's Republic of China.

\section{Conflict of interest}

None declared.

\section{Acknowlegement}

We gratefully acknowledge the technical assistance provided by Dr. Guokun Wang (Changhai Hospital, Shanghai), Dr. Yuanyuan Zhang (Zhongshan Hospital, Shanghai) and the placenta collection assistance provided by Jiayi Zhou and Jing $\mathrm{Wu}$ (Department of Tissue Bank of Obstetrics and Gynecology Hospital, Shanghai). 


\section{References}

[1] C.V. Ananth, K.M. Keyes, R.J. Wapner, Pre-eclampsia rates in the United States, 1980-2010: age-period-cohort analysis, BMJ 347 (2013) f6564.

[2] B.W. Mol, C.T. Roberts, S. Thangaratinam, L.A. Magee, C.J. de Groot, G.J. Hofmeyr, Pre-eclampsia, Lancet (2015).

[3] S.A. Mastrolia, M. Mazor, G. Holcberg, E. Leron, O. Beharier, G. Loverro, O. Erez, The physiologic anticoagulant and anti-inflammatory role of heparins and their utility in the prevention of pregnancy complications, Thromb Haemost 113(6) (2015) 1236-46.

[4] L.C. Godoi, K.B. Gomes, P.N. Alpoim, M. Carvalho, B.A. Lwaleed, L.M. Sant'Ana Dusse, Preeclampsia: the role of tissue factor and tissue factor pathway inhibitor, J Thromb Thrombolysis 34(1) (2012) 1-6.

[5] J. Said, G. Dekker, Pre-eclampsia and thrombophilia, Best Pract Res Clin Obstet Gynaecol 17(3) (2003) 441-58.

[6] Y. Miyagi, H. Yasumitsu, T. Eki, S. Miyata, N. Kkawa, F. Hirahara, I. Aoki, K. Misugi, K. Miyazaki, Assignment of the human PP5/TFPI-2 gene to $7 q 22$ by FISH and PCR-based human/rodent cell hybrid mapping panel analysis, Genomics 35(1) (1996) 267-8.

[7] C.A. Sprecher, W. Kisiel, S. Mathewes, D.C. Foster, Molecular cloning, expression, and partial characterization of a second human tissue-factor-pathway inhibitor, Proc Natl Acad Sci U S A 91(8) (1994) 3353-7.

[8] S. Wang, X. Xiao, X. Zhou, T. Huang, C. Du, N. Yu, Y. Mo, L. Lin, J. Zhang, N. Ma, M. Murata, G. Huang, Z. Zhang, TFPI-2 is a putative tumor suppressor gene frequently inactivated by promoter hypermethylation in nasopharyngeal carcinoma, BMC Cancer 10 (2010) 617.

[9] Y. Xiong, Q. Zhou, F. Jiang, S. Zhou, Y. Lou, Q. Guo, W. Liang, D. Kong, D. Ma, X. Li, Changes of plasma and placental tissue factor pathway inhibitor-2 in women with preeclampsia and normal pregnancy, Thromb Res 125(6) (2010) e317-22.

[10] Q. Zhou, Y. Xiong, Y. Chen, Y. Du, J. Zhang, J. Mu, Q. Guo, H. Wang, D. Ma, X. Li, Effects of tissue factor pathway inhibitor-2 expression on biological behavior of BeWo and JEG-3 cell lines, Clin Appl Thromb Hemost 18(5) (2012) 526-33.

[11] O. Koukoura, S. Sifakis, D.A. Spandidos, DNA methylation in the human placenta and fetal growth (review), Mol Med Rep 5(4) (2012) 883-9.

[12] J. Xuan, Z. Jing, Z. Yuanfang, H. Xiaoju, L. Pei, J. Guiyin, Z. Yu, Comprehensive analysis of DNA methylation and gene expression of placental tissue in preeclampsia patients, Hypertens Pregnancy (2016) 1-10.

[13] M.R. Branco, M. King, V. Perez-Garcia, A.B. Bogutz, M. Caley, E. Fineberg, L. Lefebvre, S.J. Cook, W. Dean, M. Hemberger, W. Reik, Maternal DNA Methylation Regulates Early Trophoblast Development, Dev Cell 36(2) (2016) 152-63.

[14] T. Ribarska, M. Ingenwerth, W. Goering, R. Engers, W.A. Schulz, Epigenetic inactivation of the placentally imprinted tumor suppressor gene TFPI2 in prostate carcinoma, Cancer Genomics Proteomics 7(2) (2010) 51-60.

[15] Q. Zhang, Y. Zhang, S.Z. Wang, N. Wang, W.G. Jiang, Y.H. Ji, S.L. Zhang, Reduced expression of tissue factor pathway inhibitor-2 contributes to apoptosis and angiogenesis in cervical cancer, J Exp Clin Cancer Res 31 (2012) 1.

[16] P.A. Jones, S.B. Baylin, The fundamental role of epigenetic events in cancer, Nat Rev Genet 3(6) (2002) 415-28. 
[17] J. Rollin, S. lochmann, C. Blechet, F. Hube, S. Regina, S. Guyetant, E. Lemarie, P. Reverdiau, Y. Gruel, Expression and methylation status of tissue factor pathway inhibitor-2 gene in non-small-cell lung cancer, Br J Cancer 92(4) (2005) 775-83.

[18] G.J. Wang, Y.B. Wang, D.N. Li, C. Li, B.B. Deng, Expression of tissue factor pathway inhibitor-2 in gastric stromal tumor and its clinical significance, Exp Ther Med 7(2) (2014) 513-517.

[19] Y. Dong, Q. Tan, L. Tao, X. Pan, L. Pang, W. Liang, W. Liu, W. Zhang, F. Li, W. Jia, Hypermethylation of TFPI2 correlates with cervical cancer incidence in the Uygur and Han populations of Xinjiang, China, Int J Clin Exp Pathol 8(2) (2015) 1844-54.

[20] C. Ferretti, L. Bruni, V. Dangles-Marie, A.P. Pecking, D. Bellet, Molecular circuits shared by placental and cancer cells, and their implications in the proliferative, invasive and migratory capacities of trophoblasts, Hum Reprod Update 13(2) (2007) 121-41.

[21] Hypertension in pregnancy. Report of the American College of Obstetricians and Gynecologists' Task Force on Hypertension in Pregnancy, Obstet Gynecol 122(5) (2013) 1122-31.

[22] J. Pan, D. Ma, F. Sun, W. Liang, R. Liu, W. Shen, H. Wang, Y. Ji, R. Hu, X. Luo, H. Shi, Over-expression of TFPI-2 promotes atherosclerotic plaque stability by inhibiting MMPs in apoE-/- mice, Int J Cardiol 168(2) (2013) 1691-7.

[23] H. Williams, J.L. Johnson, K.G. Carson, C.L. Jackson, Characteristics of intact and ruptured atherosclerotic plaques in brachiocephalic arteries of apolipoprotein E knockout mice, Arterioscler Thromb Vasc Biol 22(5) (2002) 788-92.

[24] Y.R. Lin, H.H. Chen, Y.C. Lin, C.H. Ko, M.H. Chan, Antinociceptive actions of honokiol and magnolol on glutamatergic and inflammatory pain, J Biomed Sci 16 (2009) 94.

[25] C. Cordon-Cardo, G. Dalbagni, G.T. Saez, M.R. Oliva, Z.F. Zhang, J. Rosai, V.E. Reuter, A. Pellicer, p53 mutations in human bladder cancer: genotypic versus phenotypic patterns, Int J Cancer 56(3) (1994) 347-53.

[26] N. Fukuda, H. Tanaka, Y. Tominaga, M. Fukagawa, K. Kurokawa, Y. Seino, Decreased 1,25-dihydroxyvitamin D3 receptor density is associated with a more severe form of parathyroid hyperplasia in chronic uremic patients, J Clin Invest 92(3) (1993) 1436-43.

[27] M. Ehrich, M.R. Nelson, P. Stanssens, M. Zabeau, T. Liloglou, G. Xinarianos, C.R. Cantor, J.K. Field, D. van den Boom, Quantitative high-throughput analysis of DNA methylation patterns by base-specific cleavage and mass spectrometry, Proc Natl Acad Sci U S A 102(44) (2005) 15785-90.

[28] M. Ollikainen, K.R. Smith, E.J. Joo, H.K. Ng, R. Andronikos, B. Novakovic, N.K. Abdul Aziz, J.B. Carlin, R. Morley, R. Saffery, J.M. Craig, DNA methylation analysis of multiple tissues from newborn twins reveals both genetic and intrauterine components to variation in the human neonatal epigenome, Hum Mol Genet 19(21) (2010) 4176-88.

[29] S.D. Konduri, A. Tasiou, N. Chandrasekar, J.S. Rao, Overexpression of tissue factor pathway inhibitor-2 (TFPI-2), decreases the invasiveness of prostate cancer cells in vitro, Int J Oncol 18(1) (2001) 127-31.

[30] Y. Ran, J. Pan, H. Hu, Z. Zhou, L. Sun, L. Peng, L. Yu, J. Liu, Z. Yang, A novel role for tissue factor pathway inhibitor-2 in the therapy of human esophageal carcinoma, Hum Gene Ther 20(1) (2009) 41-9.

[31] C.M. Wong, Y.L. Ng, J.M. Lee, C.C. Wong, O.F. Cheung, C.Y. Chan, E.K. Tung, Y.P. Ching, I.O. Ng, Tissue factor pathway inhibitor-2 as a frequently silenced tumor suppressor gene in hepatocellular carcinoma, Hepatology 45(5) (2007) 1129-38.

[32] D. Monk, A. Wagschal, P. Arnaud, P.S. Muller, L. Parker-Katiraee, D. Bourc'his, S.W. Scherer, R. Feil, 
P. Stanier, G.E. Moore, Comparative analysis of human chromosome $7 q 21$ and mouse proximal chromosome 6 reveals a placental-specific imprinted gene, TFPI2/Tfpi2, which requires EHMT2 and EED for allelic-silencing, Genome Res 18(8) (2008) 1270-81.

[33] B.J. van den Born, J.J. Beutler, C.A. Gaillard, A. de Gooijer, A.H. van den Meiracker, A.A. Kroon, Dutch guideline for the management of hypertensive crisis -- 2010 revision, Neth J Med 69(5) (2011) 248-55.

[34] O. Erez, R. Romero, D. Hoppensteadt, N.G. Than, J. Fareed, S. Mazaki-Tovi, J. Espinoza, T. Chaiworapongsa, S.S. Kim, B.H. Yoon, S.S. Hassan, F. Gotsch, L. Friel, E. Vaisbuch, J.P. Kusanovic, Tissue factor and its natural inhibitor in pre-eclampsia and SGA, J Matern Fetal Neonatal Med 21(12) (2008) 855-69.

[35] L.M. Dusse, M.G. Carvalho, K. Getliffe, D. Voegeli, A.J. Cooper, B.A. Lwaleed, Total plasma tissue factor pathway inhibitor levels in pre-eclampsia, Clin Chim Acta 388(1-2) (2008) 230-2.

[36] M. Ogawa, S. Yanoma, Y. Nagashima, N. Okamoto, H. Ishikawa, A. Haruki, E. Miyagi, T. Takahashi, F. Hirahara, Y. Miyagi, Paradoxical discrepancy between the serum level and the placental intensity of PP5/TFPI-2 in preeclampsia and/or intrauterine growth restriction: possible interaction and correlation with glypican-3 hold the key, Placenta 28(2-3) (2007) 224-32.

[37] E. Chinni, D. Colaizzo, G.L. Tiscia, P. Martinelli, G.M. Maruotti, M. Matteo, M. Margaglione, E. Grandone, Markers of haemostasis and angiogenesis in placentae from gestational vascular complications: impairment of mechanisms involved in maintaining intervillous blood flow, Thromb Res 125(3) (2010) 267-71.

[38] J. Ekstrand, A. Razuvaev, L. Folkersen, J. Roy, U. Hedin, Tissue factor pathway inhibitor-2 is induced by fluid shear stress in vascular smooth muscle cells and affects cell proliferation and survival, J Vasc Surg 52(1) (2010) 167-75.

[39] F. Hube, P. Reverdiau, S. lochmann, J. Rollin, C. Cherpi-Antar, Y. Gruel, Transcriptional silencing of the TFPI-2 gene by promoter hypermethylation in choriocarcinoma cells, Biol Chem 384(7) (2003) 1029-34.

[40] N. Sato, A.R. Parker, N. Fukushima, Y. Miyagi, C.A. lacobuzio-Donahue, J.R. Eshleman, M. Goggins, Epigenetic inactivation of TFPI-2 as a common mechanism associated with growth and invasion of pancreatic ductal adenocarcinoma, Oncogene 24(5) (2005) 850-8.

[41] S.D. Konduri, K.S. Srivenugopal, N. Yanamandra, D.H. Dinh, W.C. Olivero, M. Gujrati, D.C. Foster, W. Kisiel, F. Ali-Osman, S. Kondraganti, S.S. Lakka, J.S. Rao, Promoter methylation and silencing of the tissue factor pathway inhibitor-2 (TFPI-2), a gene encoding an inhibitor of matrix metalloproteinases in human glioma cells, Oncogene 22(29) (2003) 4509-16.

[42] P.A. Jones, The DNA methylation paradox, Trends Genet 15(1) (1999) 34-7.

[43] T.H. Bestor, The DNA methyltransferases of mammals, Hum Mol Genet 9(16) (2000) 2395-402.

[44] Y. Gao, Z. He, Z. Wang, Y. Luo, H. Sun, Y. Zhou, L. Huang, M. Li, Q. Fang, S. Jiang, Increased expression and altered methylation of HERVWE1 in the human placentas of smaller fetuses from monozygotic, dichorionic, discordant twins, PLoS One 7(3) (2012) e33503.

[45] B. Novakovic, N.C. Wong, M. Sibson, H.K. Ng, R. Morley, U. Manuelpillai, T. Down, V.K. Rakyan, S. Beck, S. Hiendleder, C.T. Roberts, J.M. Craig, R. Saffery, DNA methylation-mediated down-regulation of DNA methyltransferase-1 (DNMT1) is coincident with, but not essential for, global hypomethylation in human placenta, J Biol Chem 285(13) (2010) 9583-93. 
Table 1. Clinical characteristics of the study population

\begin{tabular}{|c|c|c|c|}
\hline Characteristics & $\operatorname{PE}(n=19)$ & $\mathrm{NP}(\mathrm{n}=10)$ & $P$-value \\
\hline Maternal age (years) & $31.9 \pm 2.6$ & $29.6 \pm 4.60$ & 0.08 \\
\hline BMI $\left(\mathrm{kg} / \mathrm{m}^{2}\right)$ & $27.9 \pm 2.3$ & $26.2 \pm 2.0$ & 0.07 \\
\hline Gestational age (weeks) & $35.71 \pm 1.95$ & $38.98 \pm 0.78$ & 0.003 \\
\hline Systolic BP (mmHg) & $155.16 \pm 11.00$ & $120.56 \pm 12.78$ & $<0.001$ \\
\hline Diastolic BP (mmHg) & $96.74 \pm 10.54$ & $76.78 \pm 8.24$ & $<0.001$ \\
\hline Proteinuria (g/24h) & $3.23 \pm 2.39$ & 0 & $<0.001$ \\
\hline Infant birthweight (g) & $2347.11 \pm 579.59$ & $3446.67 \pm 275.04$ & $<0.001$ \\
\hline 5' appgar score & 9 & 9 & - \\
\hline
\end{tabular}

All results presented as mean $\pm \mathrm{SD}$.

* Obtained using the Mann-Whitney $U$-test on SPSS software. 


\section{Figure legends}

Figure 1 Expression and localization of TFPI-2 in PE Vs NP patients. A, Representative images of immunostaining of TFPI-2 in placenta lesions from 19 PE patients and 10 NP placentas. TFPI-2 was mainly expressed in the cytoplasm of the syncytiocytotrophoblast layers and stronger staining could be detected in PE group. Original magnification is 400 fold . Scale bars $(100 \mu \mathrm{m})$ are shown in each image. B, Quantification of TFPI-2 expression were analyzed using the software of Image-Pro ${ }^{\circledR}$ Plus. The expression of TFPI-2 was significantly increased in PE placentas than that in NP group. C, Spearman Rank correlation analysis showed a significant association between MAP and TFPI-2 expression levels. Further confirmation of TFPI- 2 mRNA and protein expression is shown. D, The relative mRNA expression of TFPI-2 gene was evaluated by qRT-PCR with GAPDH as endogenous control. E and F, TFPI-2 were determined using western blot with GAPDH as a loading control. The representative images of western blot of TFPI- 2 are shown. The relative quantity of the TFPI-2 expression were both consistent with IHC, suggesting over-expression of TFPI-2 in PE patients than that in NP group. All experiments were repeated three times at least. The mean values are shown and the data is presented as the mean $\pm \mathrm{SD}$. $* * * P<0.0001, * * P<0.01, * P<0.05$

Fig 2 MassARRAY results for TFPI-2 methylation analysis in 10 PE placentas and 10 NP placentas. A, The blue column represents the distribution of the $\mathrm{CpG}$ sites examined in the TFPI-2 gene in the schematic diagram. The studied sequence represents a 368 base pair fragment (-272 to $96 \mathrm{bp}$, relative to the TSS) of TFPI-2 gene. The different methylation levels at each site are shown. Red signal presented in both PE and NP placentas, suggesting hypomethylation of TFPI-2 
promoter in the placenta. B, The mean methylation level was analyzed between groups. The methylation level of TFPI-2 was significantly decreased in PE placentas. C, Mean methyaltion levels of $12 \mathrm{CpG}$ units in TFPI-2 gene between the PE patients and the NP placentas are shown. D-F, The methylation status at $\mathrm{CpG} \_6, \mathrm{CpG}_{-} 15$ and $\mathrm{CpG} \_18.19$ were significantly decreased in PE patients than that in NP group (The methylation status at CpG_6 in one case and CpG_15 in another case was not analyzed for unreliable data). TSS, translation start site. $* * P<0.01, * P<0.05$.

Fig 3 Expression of DNMTs in PE and NP patients using IHC. A, Representative images of immunostaining of DNMT-1 staining in placentas lesions from 19 PE patients and 10 NP placentas. DNMT-1was mainly expressed in the nuclei of the syncytiocytotrophoblast layers. Original magnification is 400 fold. Scale bars $(100 \mu \mathrm{m})$ are shown in each image. B, Quantification of DNMT-1 expression was analyzed using the percentage of staining as described aforementioned. The expression of DNMT-1 didn't show significant difference between groups (P>0.05). C, DNMT3a was faintly expressed in either PE placentas or normal placentas. D, DNMT3b was faintly or negatively expressed in either PE placentas or normal placentas. 


\section{A}
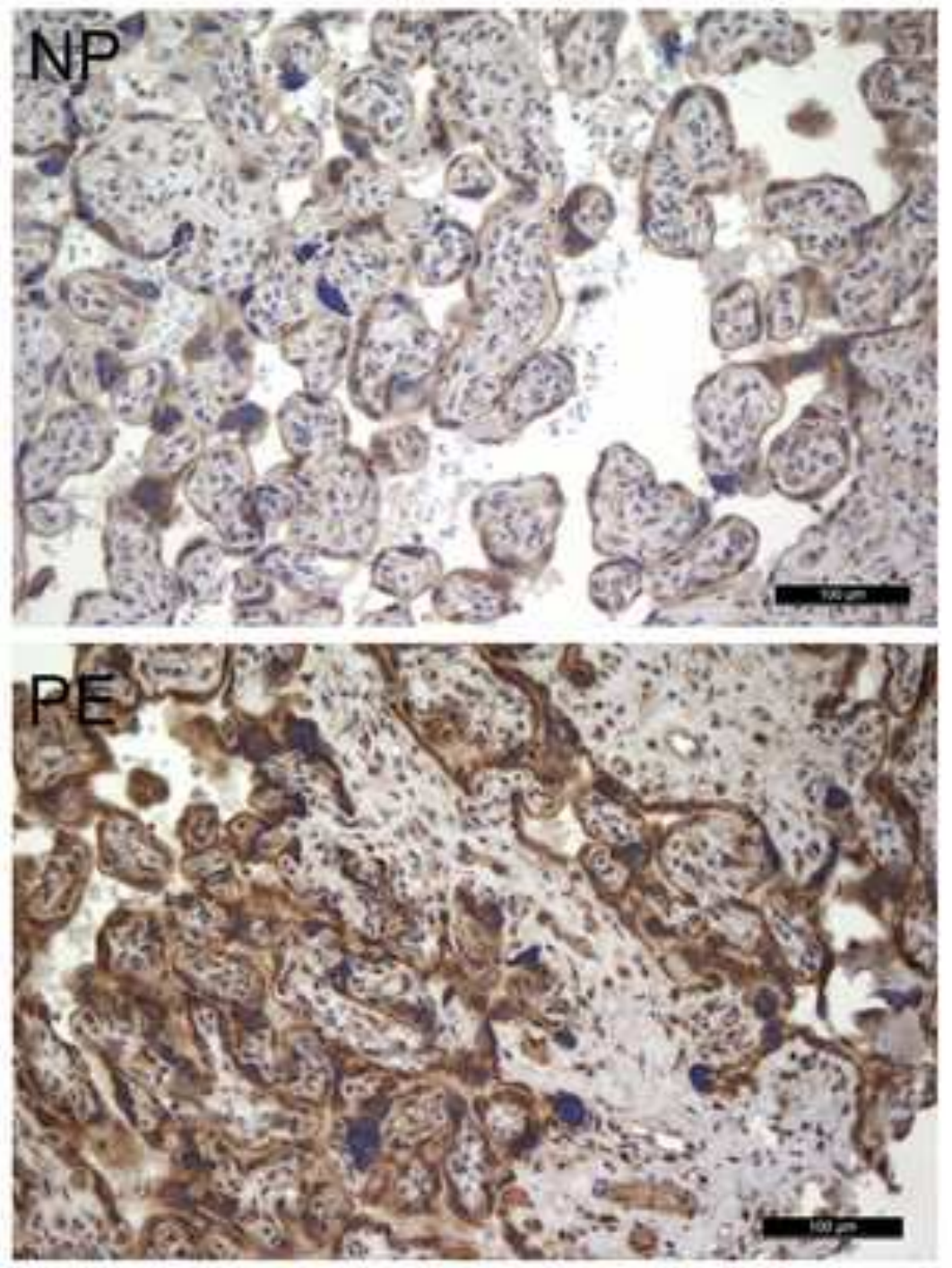

B

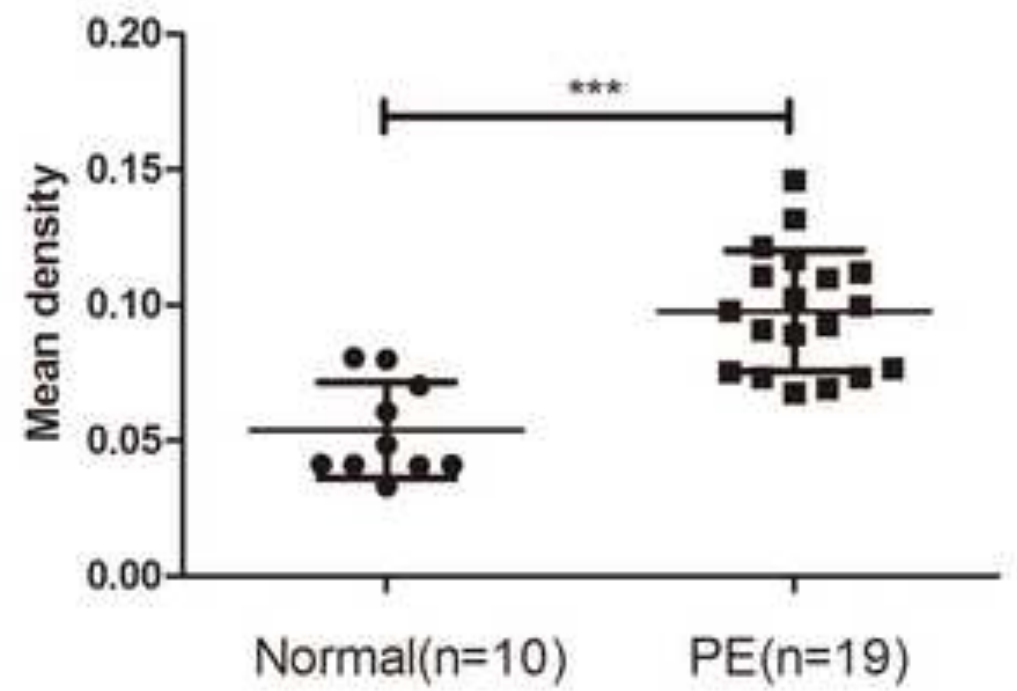

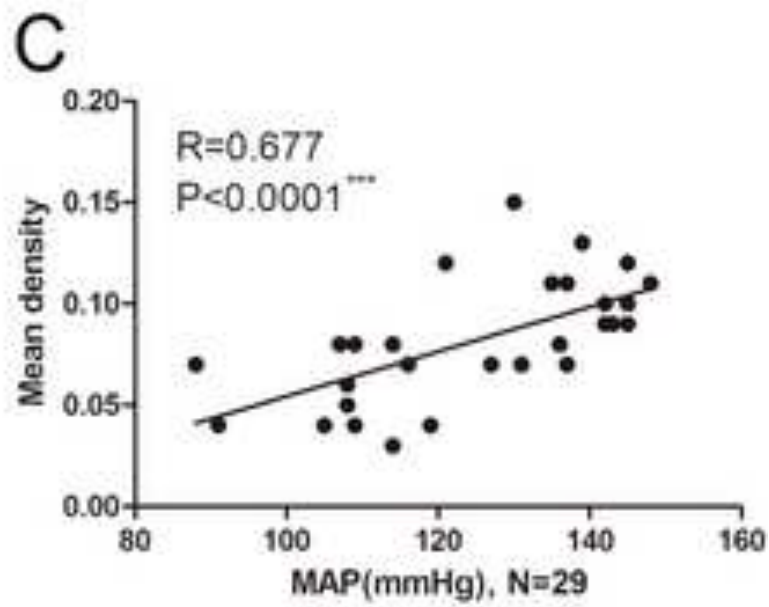

D
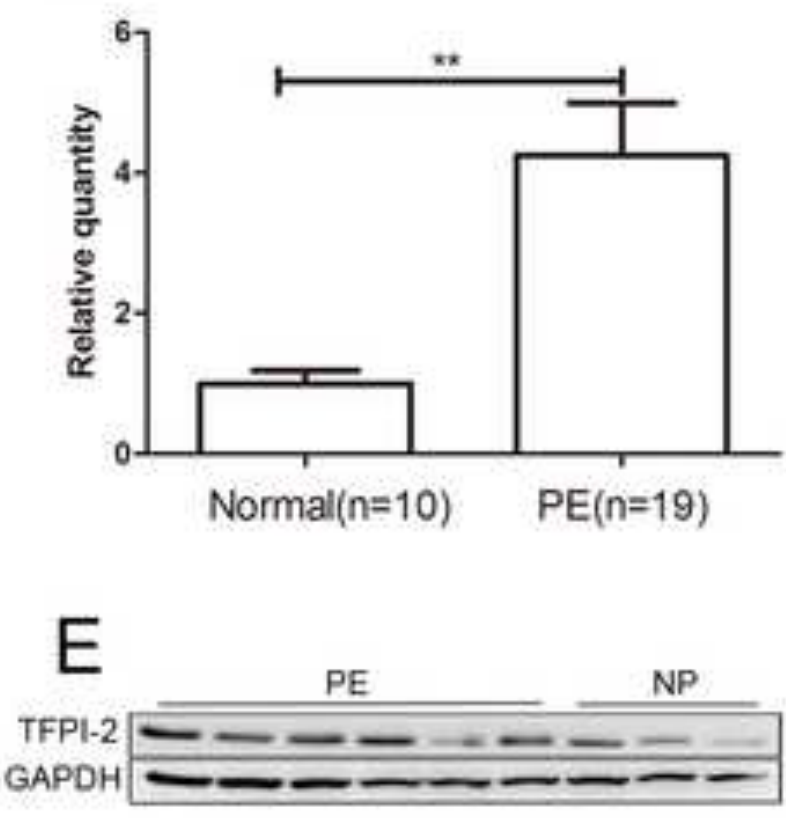

F

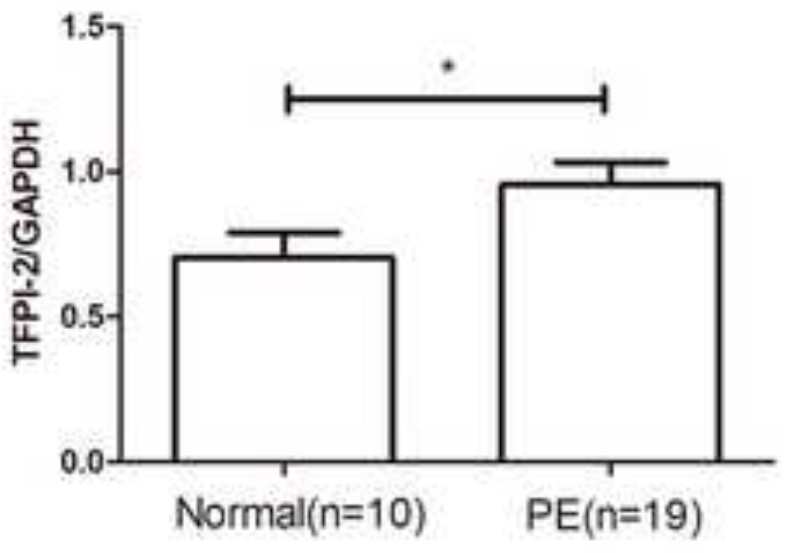



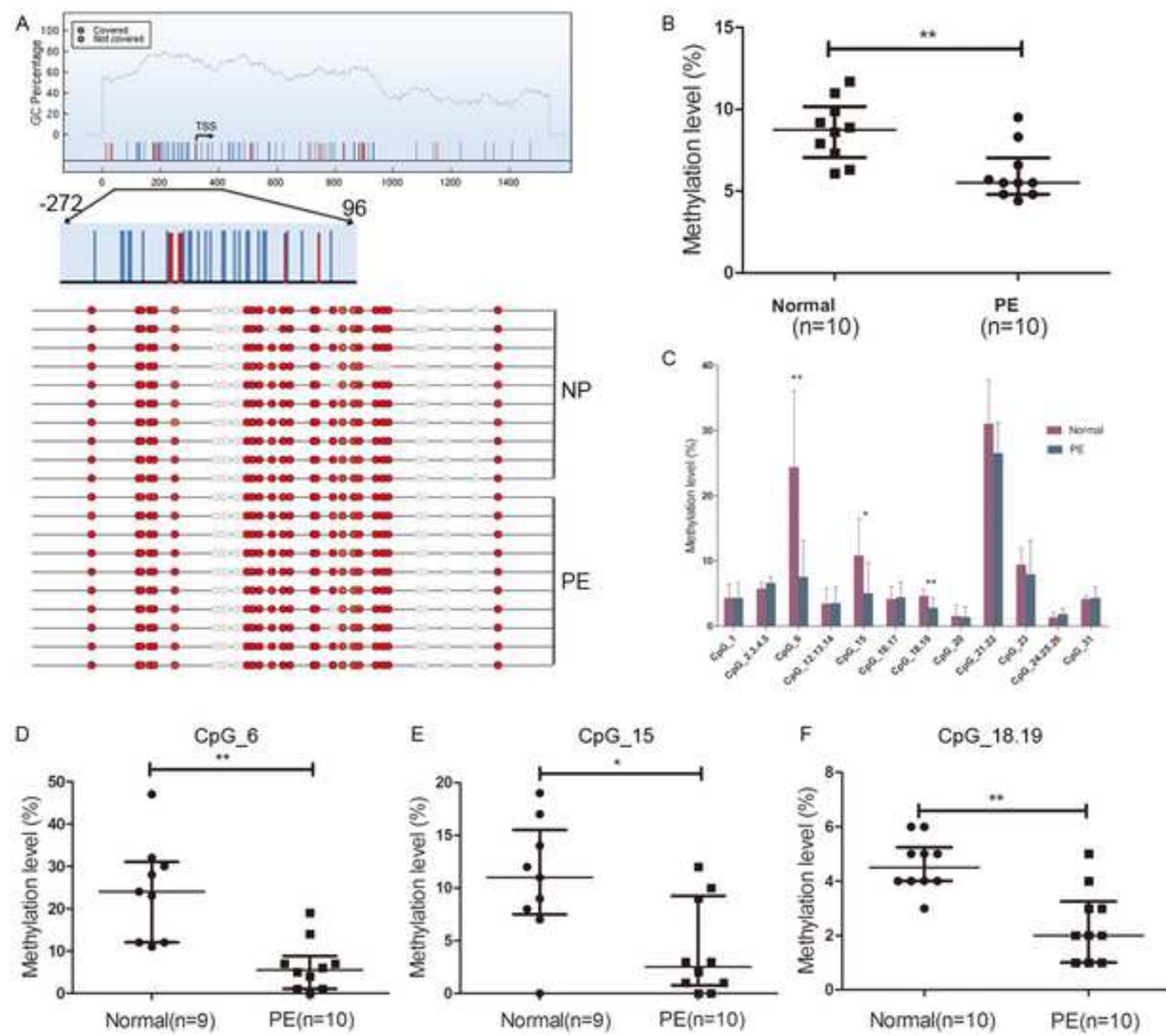
A NP

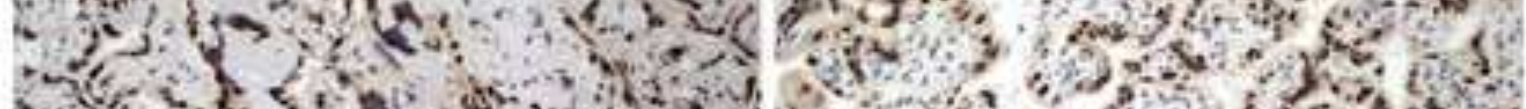

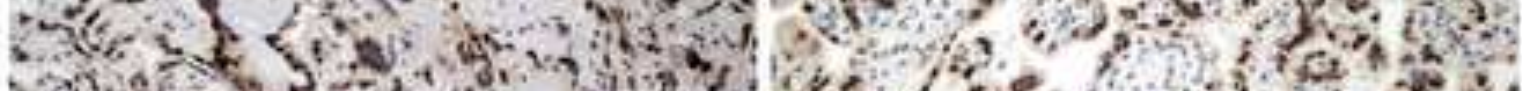

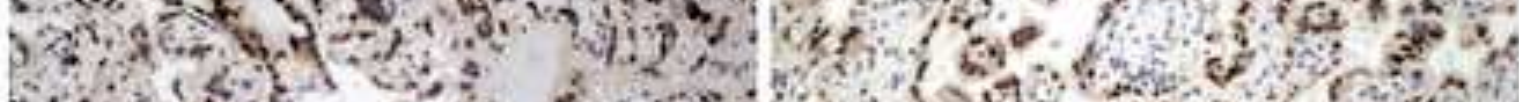

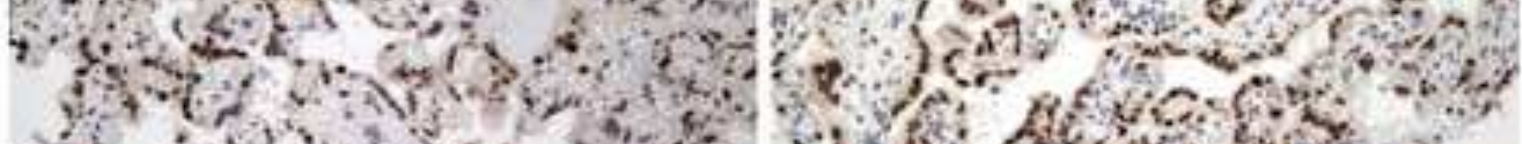

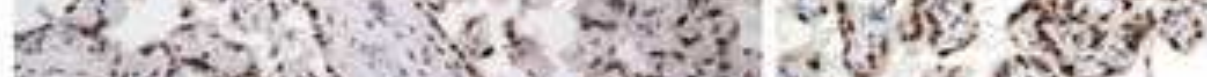
Every ty
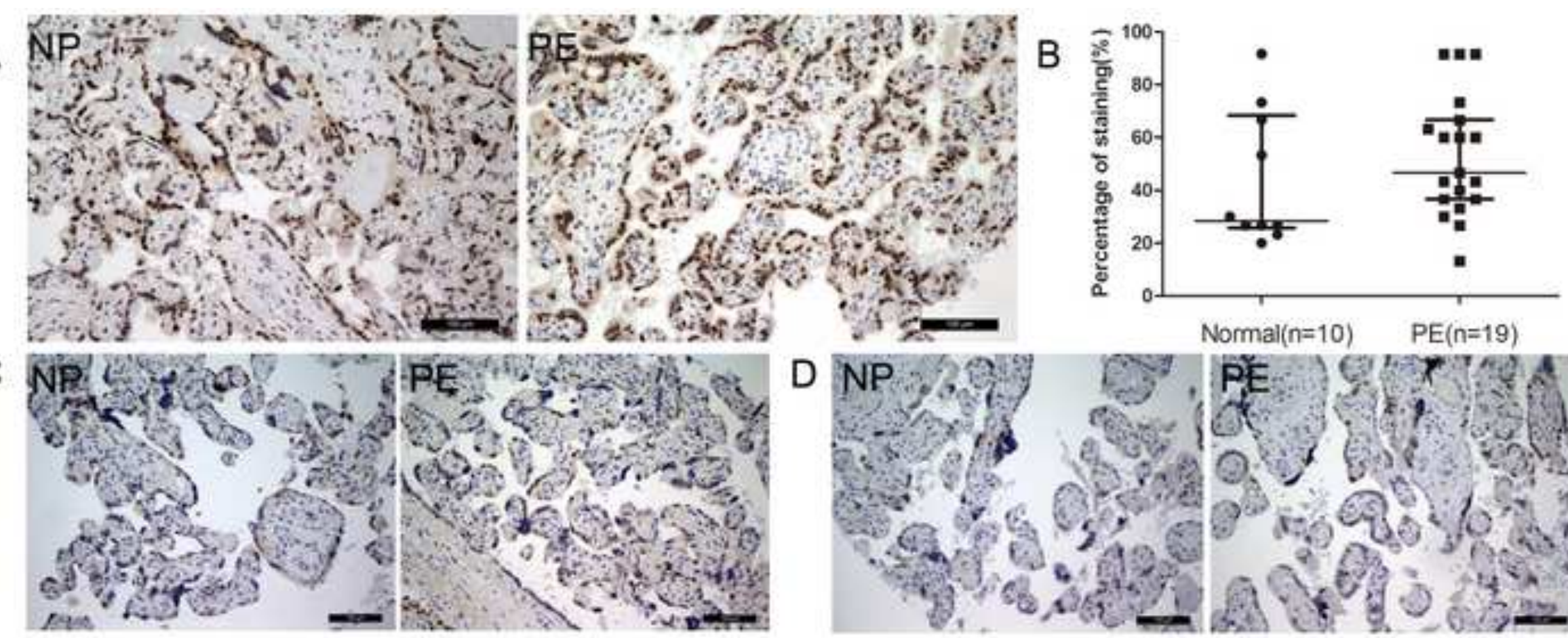

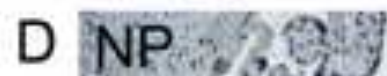

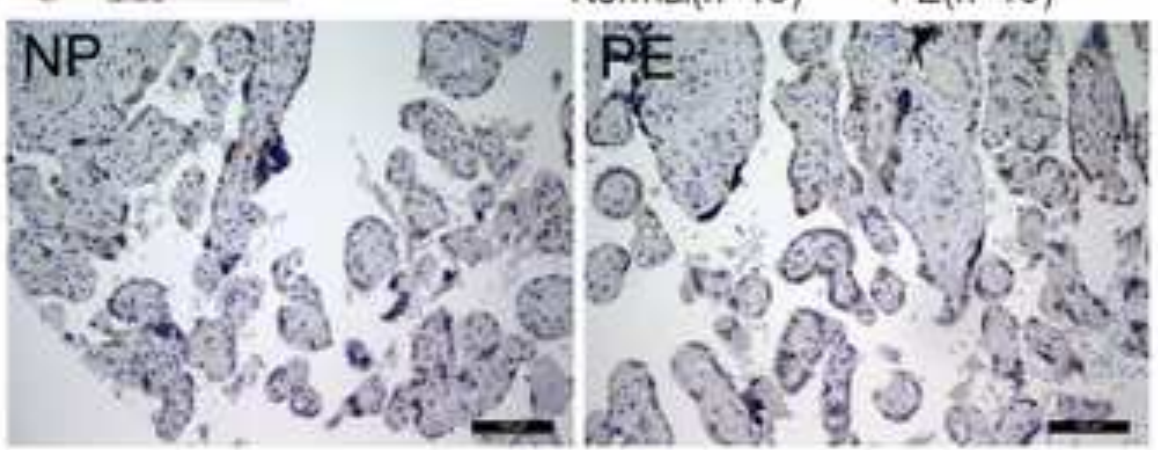

